\title{
FreeRTOS BASED ENVIRONMENTAL DATA ACQUISITION USING ARM CORTEX M4F CORE
}

\author{
Yogita Patil ${ }^{1}$, Sidharth Bhatia ${ }^{2}$ \\ ${ }^{I}$ M.Tech. (Embedded Systems), EECE Department, ITM University, Gurgaon, Haryana, India \\ ${ }^{2}$ Assistant Professor, EECE Department, ITM University, Gurgaon, Haryana, India
}

\begin{abstract}
In today's decade of Big Data and complex computing, the Data acquisition systems hold a prime position. In this project, we present a Data acquisition system, which runs on ARM Cortex M4F core microcontroller and this whole system is managed by a real time operating system names FreeRTOS. Tiva C Series Launchpad is used as the board. The system is currently designed to monitor only temperature. A temperature sensor will be used for monitoring temperature. The data received will be stored in $S D$ card. The main objective of this project is to design a data acquisition system, which is portable, cheaper and deterministic. Currently available systems in market are expensive and bulky whereas this FreeRTOS based Data acquisition system is cheaper and portable as well.
\end{abstract}

Keywords: FreeRTOS, ARM Cortex M4F core, Temperature sensor, Tiva C series Launchpad, SD card.

\section{INTRODUCTION}

The field of embedded systems is vast and it's getting broader day by day. In today's era of technology, where we have got lots and loads of data, acquisition and managing this data is also a big concern. There is unending need for data acquisition system [1]. Managing such a vast and enormous data is in itself a big task. So, such a system is required for data acquisition which is first of all easy to use, portable and cheap [2]. This paper gives a vivid description of a system based on ARM [3][4][5] having Cortex M4F core which is run by FreeRTOS. As an RTOS runs this system [6], so task scheduling becomes possible and easier. This system also has a provision to store data in SD card, which can be used further for review. The paper is split into various sections. The second section gives the basic concept of the project. Hardware and software specifications are covered in third section. Block diagram falls under fourth section. Screen shot of results is shown in fifth section. Sixth and seventh section deals with application areas and advantages. Eighth section entails the conclusion along with future work.

\section{BASIC CONCEPT}

The prime component in this project is the Tiva C Series Launchpad. This Launchpad contains TM4C123GH6PM microcontroller. The TM4C123GH6PM microcontroller has ARM Cortex M4F [7] core. There is an inbuilt temperature sensor [8] on the board, Tiva C Series Launchpad. This temperature sensor on the PE3 pin helps to acquire precisely environmental temperature [9][10]. RTOS is ported on the microcontroller. Here, we have used FreeRTOS, as it is open-source. So, FreeRTOS [11] is ported on to TM4C123GH6PM microcontroller. Then FreeRTOS is programmed for carrying out temperature acquisition task [12]. Booster pack for SD card is interfaced on the launchpad to interface the SD card. The data received is stored onto SD card.

\section{HARDWARE AND SOFTWARE DETAILS}

There are always some Hardware and Software tools required for a project. The different hardware and software used in this project are listed as below:

\subsection{Hardware Tools}

- Tiva C Series Launchpad

- Temperature Sensor

- $\quad$ SD card Booster pack

\subsection{Software Tools}

- $\quad$ CCS (Code Composer Studio version5.4)

- $\quad$ FreeRTOS version8.0.1 (RTOS)

- $\quad$ Microsoft Visual Studio 2010

\section{BLOCK DIAGRAM}

The block diagram given below explains the whole process of the system. The Tiva C Series Launchpad is the prime component in this project. Tiva Launchpad is an ARM based board. The microcontroller used in this kit is TM4C123GH6PM which has ARM Cortex M4F core. FreeRTOS is ported onto the microcontroller. FreeRTOS helps in scheduling the temperature acquisition task according to its priorities. So, it actually acts as an operating system for the real time system. The temperature sensor is inbuilt in this kit, it is at PE3 pin. It will sense the temperature and give the data to FreeRTOS for scheduling. The data acquired will be then redirected to SD card. This $\mathrm{SD}$ card helps to store data acquired from sensor. 


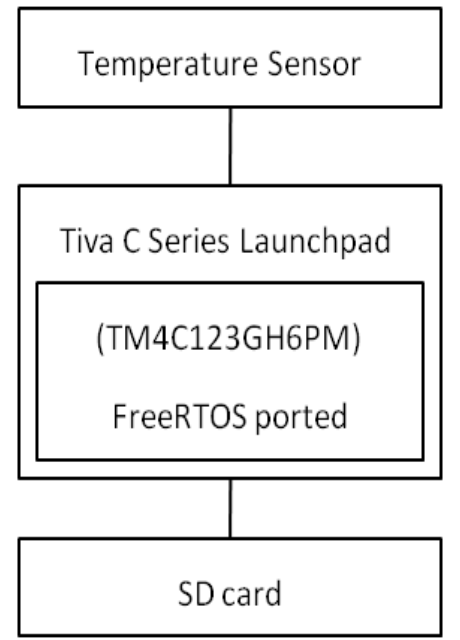

Fig -1: Block Diagram

\subsection{Tiva C Series Launchpad}

Tiva C Series Launchpad is from the makers of Texas Instruments. It is an improved version to the Stellaris Launchpad. They are cheap, self-contained, single-board microcontrollers, which features an ARM Cortex-M4F 32bit core. Launchpad provides top performance and advanced integration. There are many input-output pins that have dual functionality, which means that they can be easily be configured as digital inputs or outputs, providing access to variety of applications. The LaunchPad's TM4C123GH6PM ARM Cortex M4F 32-bit microcontroller is capable of fuelling up to $80 \mathrm{MHz}$. The controller can be run at lower speed to reduce energy consumption. It has many features, which makes it a worth choice.

\subsection{FreeRTOS}

FreeRTOS is an open source RealTime Operating system. Its design is developed in such a way that it fits on very small embedded systems and implements very minimalist set of functions. It has many features, which makes it a good choice to opt for. Amongst the various features, some of them are as follows: preemptive tasks, a small footprint written in $\mathrm{C}$ and compiled with various $\mathrm{C}$ compiler. It also provides a provision to implement mutex binary and counting semaphores and queues. FreeRTOS supports unlimited number of tasks to be run as far as hardware can handle it and of course memory too. There are many functions available in FreeRTOS. The entire life cycle of a task in FreeRTOS can be explained as follows. The "not running" state can further be categorized as ready, blocked and suspended. A task which is blocked can be changed to suspended. A task which is running can be suspended. By using different functions, one can switch between the not running state and resume the function which the user wants. The main aim of Task scheduling is to decide which task is in "Ready" state which has to be run at a given time. FreeRTOS achieves this purpose with priorities given to tasks when they are created. Priority of a task is the prime element the scheduler takes into account to decide which task has to be switched in or switched out.

\subsection{SDcard}

SD card is used in this project, to store the acquired data [13]. When the SD card is formatted, file system will be written into flash region. So, this is the region where the file system exists. 'SD card controller' helps to communicate 'main core' with the external devices. It responds to a certain set of standard SD commands and read/write data from the memory core in [14]. SD card is interfaced with the microcontroller using serial data bus. It is connected using 'SD buses'. The 'SD bus' is particularly designed for high speed whereas the SPI bus can operate with much lower speed only. The microcontroller can read/write data to the memory core and read the registers using standard SD commands send through these serial buses [15]. Before the memory card starts responding to the commands, the memory card should be triggered. Certain set of commands should be shooted one after other to commence the SD card. The SD card will switch to SD interfacing mode on reset.

\section{PROCESS FLOW}

The first and foremost task will be data acquisition. The environmental temperature will be acquired by temperature sensor. This temperature sensor will send the data to ADC. The microcontroller TM4C123GH6PM on Tiva C Series Launchpad, has 12 bit ADC in it. Now, FreeRTOS ported on TM4C123GH6PM takes the lead. FreeRTOS will schedule the temperature task and the resulting acquired data will be written onto SD card. So, the temperature is written onto SD card. This is how, the entire process flow takes place.

\section{RESULT}

Precise results have been obtained in laboratory by following the above process flow. Figure 2 shows the FreeRTOS ported onto Launchpad. The red LED shown in the snapshot blinks after every 6 seconds. Figure 3 shows the Launchpad interfaced with SD card. Booster packs for SD card is used. Figure 4 shows the temperature reading acquired in Code Composer Studio. It displays the temperature in Celsius as well as Fahrenheit.

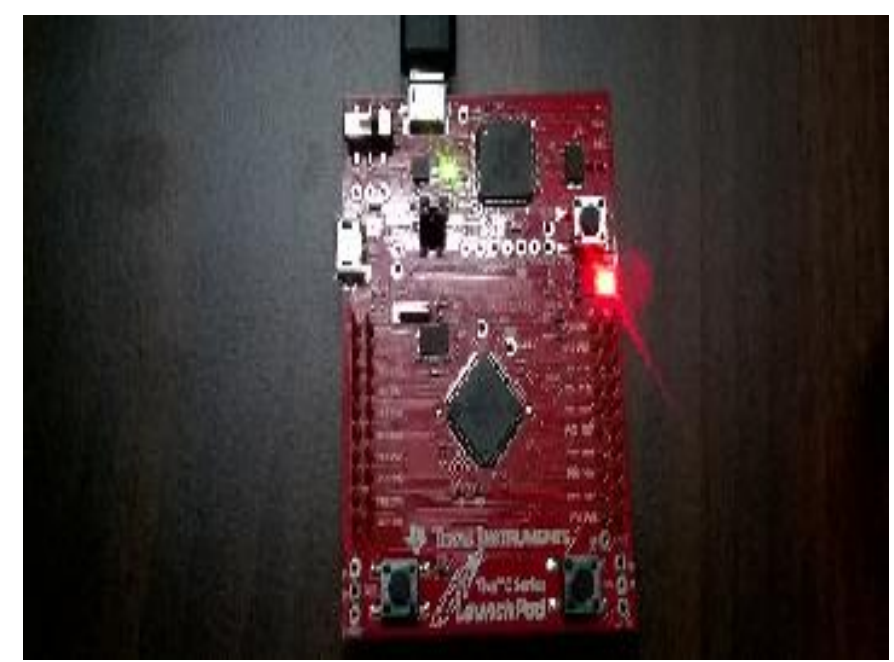

Fig -2: FreeRTOS ported on Launchpad 


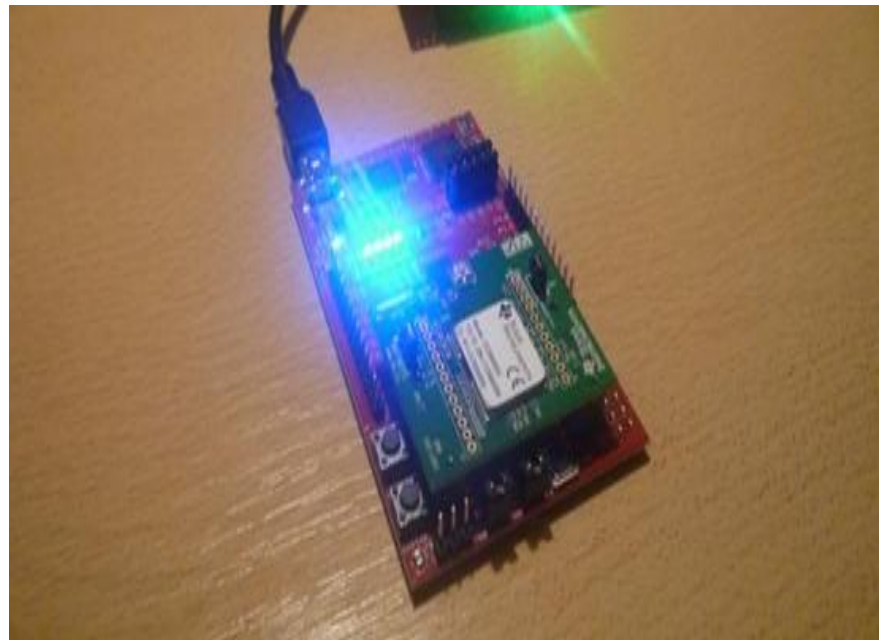

Fig -3: Screenshot of Launchpad interfaced with SD card

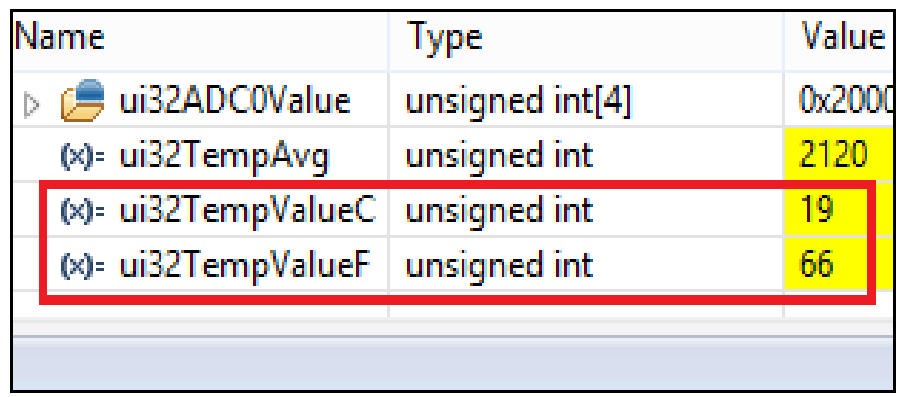

Fig -4: Screenshot of obtained simulated results in CCS

\section{APPLICATION AREAS}

FreeRTOS (Free Real Time Operating System) based Data Acquisition system can be used for environmental data capturing. It can also be used for monitoring certain parameters like Temperature, Humidity and Motion in industries. Currently it is optimized to monitor just temperature. It can be of profound use in cryogenic industries for low temperature monitoring. This project can be of utmost use in industrial applications. As it has the capacity to store data, so this data can further be analyzed in matlab to monitor the green house effect.

\section{ADVANTAGES}

The first and foremost advantage of this system is, it is portable and cheap. It is light weight, unlike other systems in market. It has data storage facility, which is another plus point. This Data acquisition system runs on FreeRTOS, which makes it a real robust system. The scheduling of task is done at its best, which makes the system void of time delays.

\section{CONCLUSION AND FUTURE SCOPE}

There are ample of Data acquisition systems available in the market, but we needed to design one which is cheaper than most of them, portable and robust. So, we have successfully made this system which is much cheaper and it uses ARM Cortex M4F core i.e. TM4C123GH6PM microcontroller, which is the latest and much efficient microcontroller. It also has a facility to store data in a SD card which is worth it. This project can be further enhanced by adding different types of sensors like Humidity sensor and Motion sensor. The acquired data can be sent on to the cloud and from there using AMQP protocol, data can be accessed. Diverting data on to server or cloud would help to increase the accessibility of the data for the analysts or users.

\section{ACKNOWLEDGEMENTS}

I would like to express my gratitude towards my mentor, who always supported and encouraged me to do the things, which i thought were impossible. I am highly obliged for his guidance. I would also like to thank the entire faculty and lab technicians of ITM University, Gurgaon. Again vote of thanks for all the authors, whose work was helpful for me.

\section{REFERENCES}

[1]. Daogang Peng, Hao Zhang, Kai Zhang, Hui Li1, Fei Xia, "Research and Development of the Remote I/O Data Acquisition System Based on Embedded ARM Platform" 2009 IEEE

[2]. Jiangchun Xu, Jiande Wu, Yuhui Li , “A Networks Data Collection Embedded System Based on ARM-uCLinux" 2009 IEEE

[3]. Hua Fang, Ming Tang, Lian Peng "Wireless data acquisition system based on ARM" 2011 IEEE

[4]. Gan-ping Li "Design of an Embedded Control and Acquisition System for Industrial Local Area Networks Based on ARM" 2010 IEEE

[5]. Yakun, Xiaodong Cheng, "Design and Implementation of Embedded Web Server Based on ARM and Linux" 2010 IEEE

[6]. Liu Zhongyuan, Cui Lili, Ding Hong, published a paper titled "Design of Monitors Based on ARM7 and Micro C/OS-II" 2010 IEEE

[7]. M.Jenko, N.Medjeral \& P. Butala, "Component-based software as a framework for concurrent design of programs and platforms-an industrial kitchen appliance embedded system," Microprocessors and Microsystems, September 2001, pp. 287-296

[8]. Peng D.G, Zhang H., Yang L, "Design and Realization of Modbus Protocol Based on Embedded Linux System". The 2008 International Conference on Embedded Softwares and Systems Symposia. July 29-31, 2008, Chengdu, Sichuan, China, pp:275-280.

[9]. Li D., Zhong C.Q., Yuan X.F. "Study on Embedded Equipment Web Monitoring and Control Based on Industrial Ethernet". Proceedings of the 6th World Congress on Control and Automation, June 21-23, 2006, Dalian, China, pg: 4600-4603

[10]. Patinge, S, Suryawanshi, Y, Kakde S, "Design of ARM based data acquisition \& control using GSM \& TCP/IP network", 2013 IEEE International Conference, 2628 Dec. 2013 Page(s): 1-4

[11]. Rafia Inam, Jukka Mäki-Turja, Mikael Sjödin, Mohammad Ashjaei, Sara Afshar: "Support for Hierarchical Scheduling in FreeRTOS", 16th IEEE International Conference on Emerging Technologies and Factory Automation 
[12]. Wenbin Huang, Guanglong Wang, Jianglei Lu, FengQi

Gao: "Research of wireless sensor networks for an intelligent measurement system based on ARM ", 2011 International Conference on Mechatronics and Automation (ICMA), Publisher IEEE

[13]. Mahzan, N.N, Shah Alam, Malaysia, Omar A.M, Mohammad Noor, S.Z, Mohd Rodzi, M.Z, "Design of data logger with multiple SD cards", 2013 IEEE Conference on Clean Energy and Technology (CEAT)

[14]. Zhenlin Lu, Jingjiao Li, Yao Zhang, "The Reading/Writing SD Card System Based on FPGA”, 2010 First International Conference on Pervasive Computing Signal Processing and Applications (PCSPA), Publisher IEEE

[15]. Elkeelany, O, Todakar, V.S, "Data Archival to SD Card Via Hardware Description Language", Publisher: Embedded Systems Letters, IEEE (Volume:3, Issue: 4 )

\section{BIOGRAPHIES}

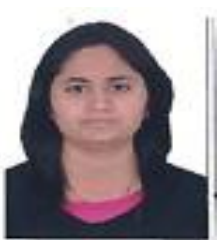

Yogita Patil is currently pursuing Masters of Technology in Embedded Systems from ITM University, Gurgaon, India. Her areas of interest are Low Power Embedded Systems, RTOS, Robotics.

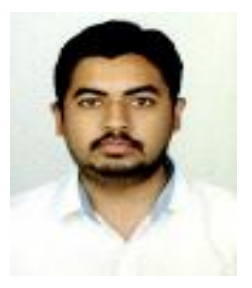

Sidharth Bhatia has received his M.Tech in Embedded Systems from SRM University, Chennai. His areas of interest are Remote sensing, Real time embedded systems, High resolution satellite image processing. He has various publications in international journals and conferences. 